\title{
On dihedral 2 - groups as inner mapping groups of finite commutative inverse property loops
}

\author{
Markku Niemenmaa
}

\begin{abstract}
We show that finite commutative inverse property loops may not have nonabelian dihedral 2 - groups as their inner mapping group.
\end{abstract}

Keywords: Loop, dihedral group. inner mapping group

Mathematics Subject Classification: 20D10, 20N05

\section{Introduction}

If $Q$ is a loop then the mappings $L_{a}(x)=a x$ and $R_{a}(x)=x a$ are called the left and right translation. These two mappings are permutations on $Q$ for every $a \in Q$ and the permutation group $M(Q)=\left\langle L_{a}, R_{a}: a \in Q\right\rangle$ is called the multiplication group of $Q$. The stabilizer of the neutral element of $Q$ is the inner mapping group of $Q$ and we denote it by $I(Q)$. If $Q$ is a group then $I(Q)=\operatorname{Inn}(Q)$, the group of inner automorphisms of $Q$. We know that in the case of commutative groups $\operatorname{Inn}(Q)$ is always trivial whereas noncommutative groups have nontrivial inner automorphism groups. For example, there are three nonisomorphic groups of order 16, whose inner automorphism group is the dihedral group of order 8 . We also know that there exist nonassociative and noncommutative loops of order 16 whose inner mapping group is the dihedral group of order 8 (private communication from Michael Kinyon). What about the situation in finite commutative loops? In [2, p. 357 - 358], Drápal introduces a construction of finite commutative loops of order $2 m(m \geq 3)$ such that the inner mapping group $I(Q)$ is the dihedral group of order $2 m$.

A loop $Q$ is an inverse property loop if $Q$ has a unique left and right inverse $x^{-1}$ and $x^{-1}(x y)=y=(y x) x^{-1}$ for every $x, y \in Q$. The purpose of this paper is to show that in the case of finite commutative inverse property loops, nonabelian dihedral 2 - groups do not appear as inner mapping groups of these loops.

We consider only finite loops and groups in this paper. The proofs of our main theorems rely on the use of connected transversals in finite groups and 
this notion and some basic results about these transversals are explained in the following section. For basic facts about loop theory and its connections to group theory the reader is advised to consult [1] and [3].

\section{Loops and groups: some results}

Our proofs are based on the use of connected transversals in a group (this group turns out to be the multiplication group $M(Q)$ mentioned in the introduction) and therefore we shall start with a brief discussion about connected transversals and try to give some insight into the relationship between loops and groups given by this notion.

Let $G$ be a group and $H \leq G$. If $A$ and $B$ are two left transversals to $H$ in $G$ and $a^{-1} b^{-1} a b \in H$ for every $a \in A$ and for every $b \in B$, then we say that the two transversals are $H$-connected in $G$. If $A=B$, then we say that $A$ is a selfconnected transversal to $H$ in $G$. In the following lemmas and theorems we consider some basic properties of $H$-connected transversals $A$ and $B$. We denote by $H_{G}$ the core of $H$ in $G$ (it is the largest normal subgroup of $G$ contained in $H)$.

Lemma 2.1. If $H_{G}=1$, then $1 \in A \cap B$ and $A Z(G) \subseteq A$ and $B Z(G) \subseteq B$.

For the proof, see [3, p. 113] and [6, Lemma 2.3].

From lemma 2.1 it follows that if $H_{G}=1$, then $Z(G) \subseteq A \cap B$.

Lemma 2.2. If $H_{G}=1$, then $N_{G}(H)=H \times Z(G)$.

For the proof, see [3, Proposition 2.7]

Theorem 2.3. Let $H$ be a nilpotent subgroup of $G$. If $G=\langle A, B\rangle$ and $H_{G}=1$, then $H$ is subnormal in $G$ and $Z(G)>1$.

For the proof, see [5, Theorem 2.8].

Theorem 2.4. If $H$ is cyclic and $G=\langle A, B\rangle$, then $G^{\prime} \leq H$.

For the proof, see [3, Theorem 3.5]

Theorem 2.5. Let $p$ be a prime number. If $H \cong C_{p} \times C_{p}$ and $G=\langle A, B\rangle$, then $G^{\prime} \leq N_{G}(H)$.

For the proof, see [4, Lemma 4.2].

Next we prove

Lemma 2.6. Let $G=\langle A, B\rangle$. If $H$ is nilpotent and $H_{G}=1$, then the core of $H Z(G)$ in $G$ properly contains $Z(G)$. 
Proof. By lemma 2.2 and theorem 2.3, $N_{G}(H)=H \times Z(G)$ and $Z(G)>1$. If the core of $H Z(G)$ in $G$ equals $Z(G)$, then from lemma 2.2 it follows that

$$
N_{G / Z(G)}(H Z(G) / Z(G))=H Z(G) / Z(G) \times Z(G / Z(G)) .
$$

Now we write $Z(G / Z(G))=M / Z(G)$ and thus $N_{G}(H Z(G))=H M$, where $M$ is normal in $G, Z(G)$ is a proper subgroup of $M$ and $H \cap M=1$. Then $H M=C H=D H$, where $C \subseteq A$ and $D \subseteq B$. By lemma 2.1,

$$
Z(G / Z(G)) \subseteq A Z(G) / Z(G) \cap B Z(G) / Z(G) .
$$

We conclude that $M \subseteq C Z(G) \cap D Z(G)$. If $m \in M$, then $m=c z=d r$, where $c \in A, d \in B$ and $z$ and $r$ are elements from $Z(G)$. If $t \in A \cup B$, then $[t, m] \in M \cap H=1$. Thus $C_{G}(M) \geq\langle A, B\rangle=G$. But then $M=Z(G)$, a contradiction.

We shall conclude this section by establishing the relation between connected transversals and loop theory. If $A=\left\{L_{a}: a \in Q\right\}$ and $B=\left\{R_{a}: a \in Q\right\}$ are the sets of left and right translations, then $A$ and $B$ are $I(Q)$-connected transversals in $M(Q)$. Since $M(Q)$ is transitive on $Q$, it follows that the core of $I(Q)$ in $M(Q)$ is trivial. Kepka and Niemenmaa proved the following theorem in 1990 [3, Theorem 4.1].

Theorem 2.7. A group $G$ is isomorphic to the multiplication group of a loop if and only if there exist a subgroup $H$ of $G$ satisfying $H_{G}=1$ and $H$-connected transversals $A$ and $B$ such that $G=\langle A, B\rangle$.

If $Q$ is a commutative loop, then $A=B$. Furthermore, if $Q$ is a commutative inverse property loop, then $\left(L_{a}\right)^{-1}=L_{a^{-1}}$ and thus $A=A^{-1}$.

\section{Main Theorems}

In this section we consider the situation that $A=B, A=A^{-1}$ and $H$ is a nonabelian dihedral 2 - group. We first consider the case where $|H|=8$.

Theorem 3.1. Let $H \leq G$ be a dihedral group of order 8. If $A$ is a selfconnected transversal to $H$ in $G, G=\langle A\rangle$ and $A=A^{-1}$, then $H_{G}>1$.

Proof. Assume that $H_{G}=1$. From Theorem 2.3 it follows that $Z(G)>1$ and by lemma 2.6, the core of $H Z(G)$ in $G$ is equal to $K Z(G)$, where $1<K \leq H$. If $|K| \geq 4$, then $H Z(G) / K Z(G)$ is cyclic and by theorem $2.4, G^{\prime} \leq H Z(G)$. This means that $H Z(G)$ is normal in $G$. As $(H Z(G))^{\prime}=H^{\prime} \neq 1$, we get $H_{G}>1$, a contradiction.

Thus we may assume that $|K|=2$. Clearly, $K=Z(H)=H^{\prime}$. We then consider $G / K Z(G)$ and the subgroup $H Z(G) / K Z(G)$. Now $H Z(G) / K Z(G) \cong$ $C_{2} \times C_{2}$. By theorem 2.5 and lemma 2.2, 
$(G / K Z(G))^{\prime} \leq N_{G / K Z(G)}(H Z(G) / K Z(G))=H Z(G) / K Z(G) \times Z(G / K Z(G))$.

It follows that $G^{\prime} \leq H M$, where $M / K Z(G)=Z(G / K Z(G))$. Clearly, $H M$ and $M$ are normal in $G$ and $H \cap M=K$. We may also conclude that $M=C K$, where $Z(G) \subseteq C \subseteq A$. By using lemma 2.1, we get

$$
A K Z(G) / K Z(G) \cdot C K Z(G) / K Z(G) \subseteq A K Z(G) / K Z(G) .
$$

It follows that $C A \subseteq A K$. Then let $c \in C$ and $a \in A$. Now $c a=b h$, where $b \in A$ and $h \in K$. Thus $h=b^{-1} c a$ and $h^{a^{-1}}=\left(b^{-1} c a\right)^{a^{-1}}=a b^{-1} c a a^{-1}=$ $a b^{-1} a^{-1} b b^{-1} a c=\left[a^{-1}, b\right] b^{-1} c a l=\left[a^{-1}, b\right] h l$, where $l \in H$. As $A=A^{-1}$ and $K Z(G)$ is normal in $G$, we conclude that $h^{a^{-1}} \in K^{a^{-1}} \cap H \leq K Z(G) \cap H=K$. If $h \neq 1$, then $h^{a^{-1}}=h($ as $|K|=2)$ and $a \in N_{G}(K)$.

If $d \in A$, then $(c a)^{d}=(b h)^{d}$, hence ckag $=b f h^{d}=c a h^{-1} f h^{d}$, where $k \in K$ and $g, f \in H$. It follows that $h^{d}=f^{-1} h k^{a} g \in H$. This is true for every $d \in A$, so $h \in \bigcap_{d \in A} H^{d^{-1}}=H_{G}=1$.

We may now conclude that $c a=b \in A$. As $A=A^{-1}$, we also have $c^{-1} a^{-1}=$ $w \in A$. Thus $w b=c^{-1} a^{-1} c a \in H$. But then $w=b^{-1}$ and $c^{-1} a^{-1} c a=1$. This means that $c \in Z(\langle A\rangle)=Z(G)$ and $M=C K \leq K Z(G)$. But then $G^{\prime} \leq H M \leq H Z(G)$ and $H Z(G)$ is normal in $G$. Now $(H Z(G))^{\prime}=H^{\prime} \neq 1$ and thus $H_{G}>1$, a contradiction.

We shall next consider the general situation where $H$ is any nonabelian dihedral 2 - group.

Theorem 3.2. Let $H \leq G$ be a dihedral 2-group of order $2^{n}(n \geq 3)$. If $A$ is a selfconnected transversal to $H$ in $G, G=\langle A\rangle$ and $A=A^{-1}$, then $H_{G}>1$.

Proof. Assume that $G$ is a minimal counterexample. By theorem 3.1 we may assume that $n \geq 4$. As in the proof of theorem 3.1, it follows that the core of $H Z(G)$ in $G$ is equal to $K Z(G)$, where $1<K \leq H$. Likewise, we may conclude that $H Z(G) / K(Z G)$ is not cyclic. If $H Z(G) / K Z(G)$ is dihedral of order $2^{m}$ $(n>m \geq 3$ ), then the core of $H Z(G) / K Z(G)$ in $G / K Z(G)$ is not trivial, a contradiction.

Thus we may assume that $H Z(G) / K Z(G) \cong C_{2} \times C_{2}$ and $K$ is cyclic of order $2^{n-2}$, where $n \geq 4$. Let $T=\left\langle x \in K Z(G) \mid x^{2}=1\right\rangle$. As $K Z(G)$ is normal in $G$, it follows that $T$ is normal in $G$. Then consider $G / T$ and its subgroup $H T / T$. As $H T / T$ is a nonabelian dihedral 2 - group, we conclude that the core of $H T$ in $G$ properly contains $T$. We denote this core by $E$ and conclude that $E=F M$, where $F \leq K,|F| \geq 4$ and $M \leq Z(G)$ is an elementary abelian 2 group. Then $\left\langle x^{2} \mid x \in E\right\rangle$ is a nontrivial normal subgroup of $G$ contained in $H$ and thus $H_{G}>1$. The proof is complete.

By combining theorem 2.7 with theorem 3.2 we get

Theorem 3.3. Let $Q$ be a finite commutative inverse property loop. Then the inner mapping group $I(Q)$ can not be a nonabelian dihedral 2 - group. 


\section{References}

[1] Bruck,R.H., Contributions to the theory of loops, Trans. Amer. Math. Soc. 60 (1946), $245-354$.

[2] Drápal,A., A class of commutative loops with metacyclic inner mapping groups, Comment. Math. Univ. Carolin. 49,3 (2008), 357 - 382.

[3] Kepka,T., Niemenmaa,M., On multiplication groups of loops, J.Algebra 135 (1990), 112 - 122.

[4] Kepka,T., Niemenmaa,M., On connected transversals to abelian subgroups, Bull. Australian Math. Soc. 49 (1994), 121 - 128.

[5] Niemenmaa,M., Finite loops with nilpotent inner mapping groups are centrally nilpotent, Bull. Australian Math. Soc. 79 (2009), 109 - 114.

[6] Niemenmaa,M., On finite commutative IP-loops with elementary abelian inner mapping groups of order $p^{4}$, Comment. Math. Univ. Carolin. 51,4 (2010), $559-563$.

Department of Mathematical Sciences

University of Oulu, PL 3000

90014 Oulu, Finland

E-mail: markku.niemenmaa@oulu.fi 